\title{
Contaminación armónica producida por cargas no lineales de baja potencia: modelo matemático y casos prácticos
}

\section{Harmonic Pollution Produced by Low Power Nonlinear Loads: Mathematical Model and Practical Cases}

\author{
Mora-Barajas M.A. \\ Departamento de Computación, Electrónica, y Mecatrónica. \\ Escuela de Ingeniería. \\ Universidad de las Américas Puebla. \\ E-mail:marco.morabs@udlap.mx \\ Bañuelos-Sánchez P. \\ Departamento de Computación, Electrónica, y Mecatrónica. \\ Escuela de Ingeniería. \\ Universidad de las Américas Puebla. \\ E-mail:pedro.banuelos@udlap.mx
}

(Recibido: septiembre de 2006; reevaluado: enero de 2009; aceptado octubre de 2009)

\section{Resumen}

Muchos de los aparatos eléctricos que tenemos en casa como televisores, videograbadoras y computadoras, generan armónicos de voltaje y de corriente que incrementan las pérdidas de potencia y contaminan las líneas de suministro eléctrico, pudiendo ocasionar severos daños tanto a las redes de energía como a los equipos que de ellas se alimentan. En este artículo se muestra un análisis matemático del contenido armónico en la corriente de un circuito que representa a una carga residencial no lineal. Además, a partir de mediciones eléctricas realizadas de manera experimental, se presenta un estudio comparativo de la contaminación armónica que este tipo de cargas introduce a las líneas de suministro eléctrico.

Descriptores: factor de potencia, pérdidas de potencia, cargas no lineales, distorsión armónica, calidad de la energía eléctrica.

\begin{abstract}
Many household electrical appliances like televisions, video recorders and computers are sources of voltage and current harmonics, which increase power losses and contaminate the AC power line. These harmonics can cause serious problems in both, utilities and electrical equipments connected to the same $A C$ line. This paper shows a mathematical analysis of harmonics content on the electrical current for a circuit representing a non linear residential load. A comparative harmonic distortion study produced by the residential loads on the electrical power lines, measured experimentally, is also shown.
\end{abstract}

Keywords: Power factor, power losses, non-linear loads, low power, harmonic distortion, electrical energy quality. 
DOI: http://dx.doi.org/10.22201/fi.25940732e.2010.11n2.016

Contaminación armónica producida por cargas no lineales de baja potencia: modelo matemático y casos prácticos

\section{Introducción}

En los últimos años se ha incrementado la preocupación por un mejor aprovechamiento de la energía eléctrica, tanto por parte de los productores como de los usuarios. Si bien, los avances en áreas como la electrónica de potencia han permitido aumentar la eficiencia de los equipos eléctricos, éstos representan cargas no lineales que pueden deteriorar la calidad de la energía entregada por las compañías generadoras de electricidad. A pesar de que también distorsionan la onda fundamental de voltaje de $60 \mathrm{~Hz}$, la principal característica por la cual se les denomina cargas no lineales, es la gran distorsión que producen sobre la corriente que le demandan a la línea de alimentación alterna, debido a la generación inherente de armónicos. En este sentido, las cargas resistivas, inductivas y capacitivas se consideran cargas lineales, ya que demandan una corriente prácticamente sinusoidal (Abad, 1998).

Un armónico, según la norma IEEE-519, está definido como el contenido de la señal, cuya frecuencia es un múltiplo entero de la frecuencia de repetición base o frecuencia fundamental (Hibbard, 1995). Su presencia puede ocasionar una gran variedad de problemas, tanto al suministro eléctrico como a los usuarios; por ejemplo: calentamiento excesivo de los transformadores y de cables conductores (principalmente del neutro), mal funcionamiento de las plantas eléctricas así como en sistemas circundantes a los que producen la contaminación armónica, resonancias, interferencia electromagnética con equipos de comunicación, etc. (Brugnoni, 2003; Jiménez et al., 2005; Eguíluz et al., 2001).

A través de normas y estándares como la mencionada IEEE-519, se han marcado tolerancias permisibles para los niveles de contaminación armónica producida por cargas no lineales, a fin de mantener la calidad en la energía eléctrica. En la norma internacional IEC 1000-3-2, por ejemplo, se establecen los límites para los armónicos de corriente en aquellos aparatos que consumen menos de $16 \mathrm{~A}$ por fase. En este trabajo, se presenta un estudio de la contaminación armónica producida por diversas cargas residenciales, que caen precisamente dentro de esta categoría de la norma IEC 1000-3-2.

En la siguiente sección, se realiza el análisis de un circuito eléctrico empleado para modelar una carga residencial no lineal y se muestran los resultados obtenidos en simulación del contenido armónico en la corriente que se suministra al circuito. Posteriormente, se presenta un estudio de la contaminación armónica producida por diversas cargas residenciales, medida experimentalmente conforme lo establece la norma internacional (IEC 1000-3-2). Finalmente, en la última sección se dan las conclusiones.

\section{Un modelo de carga no lineal de baja potencia}

Muchos de los aparatos que tenemos en casa como televisores, consolas de video juegos, videograbadoras, y computadoras producen distorsión, tanto al voltaje como a la corriente del suministro eléctrico, pues poseen fuentes de alimentación que utilizan rectificadores de onda completa para la conversión de corriente alterna (CA) a corriente directa (Suárez et al., 2005).

De manera general, es posible representar una carga residencial no lineal a través del circuito mostrado en la figura 1. $R_{s}$ y $L_{s}$ representan la impedancia de la línea de $\mathrm{CA}$, o bien, dado que algunos aparatos emplean un transformador para reducir el voltaje de la línea, $R_{s}$ y $L_{s}$ podrían ser parámetros de dicho transformador. $L_{0}$ y $C$ constituyen el filtro para obtener un voltaje y una corriente continuos (Arrillaga et al., 1985), y $R_{0}$ representa al elemento que consume la potencia entregada a la carga no lineal. $v_{s}$ representa la línea de alimentación alterna para uso residencial $\left(v_{s}=\sqrt{2} V_{r m s} \operatorname{sen}(\omega t)\right.$; donde $V_{r m s}$ es el voltaje eficaz y $\omega=2 \pi$, siendo $f$ la frecuencia de la línea).

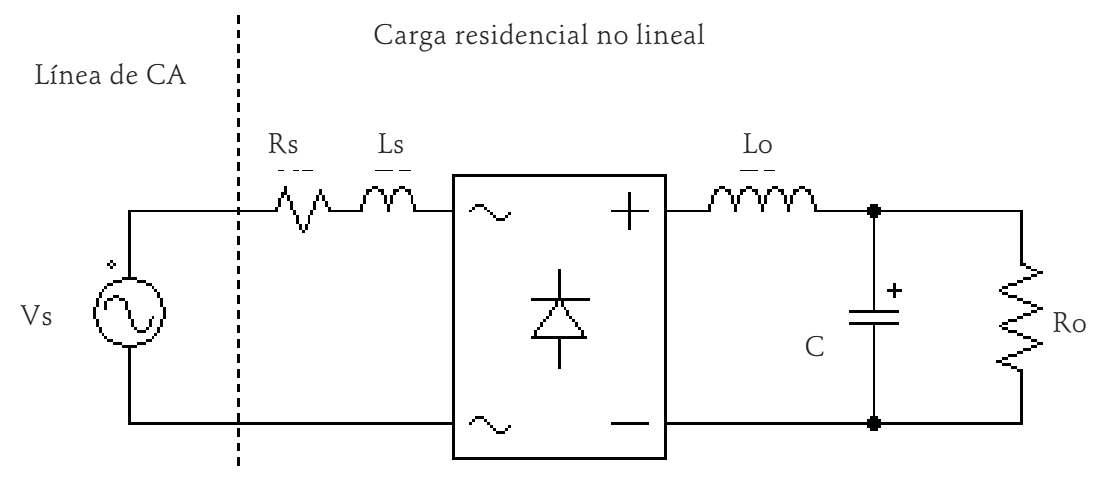

Figura 1. Modelo de una carga residencial, cuya fuente utiliza un puente rectificador 
Considerando como ideales los componentes del circuito, durante el semiciclo positivo del voltaje de entra$\mathrm{da}, v_{s}$, conducirán un par de diodos del puente rectificador, el segundo par de diodos entrará en conducción durante el semiciclo negativo. Este comportamiento del puente rectificador, permite representar el circuito de la figura 1 como se muestra en la figura 2 , mientras exista un par de diodos conduciendo. Dado que $L_{s}$ queda en serie con $L_{0}$, podemos considerar un inductor equivalente, de valor $L=L_{s}+L_{0}$.

Aplicando las leyes de Kirchhoff es posible obtener que:

$$
\begin{aligned}
& v_{0}=v_{s}-R_{S} i_{s}-L \frac{d i_{s}}{d t} \\
& i_{s}=C \frac{d v_{0}}{d t}+\frac{1}{R_{0}} v_{0}
\end{aligned}
$$

Sustituyendo (1) en (2), se tiene que:

$$
\frac{d i_{s}^{2}}{d t^{2}}+2 \alpha \frac{d i_{s}}{d t}+\omega_{n}^{2} i_{s}=\frac{1}{L}\left(\frac{d v_{s}}{d t}+\frac{1}{R_{o} C} v_{s}\right)
$$

siendo

$$
\alpha=\frac{1}{2}\left(\frac{R_{s}}{L}+\frac{1}{R_{0} C}\right) ; \omega_{n}=\sqrt{\frac{R_{s}+R_{0}}{R_{o} L C}}
$$

La solución completa de la ecuación (3) tiene dos partes, una componente natural y una forzada. La respuesta forzada está dada por:

$$
i_{f}(t)=\frac{V_{m}}{Z_{i}} \operatorname{sen}\left(\omega t-\theta_{i}\right)
$$

donde,

$$
Z_{i}=\frac{z}{\sqrt{1+\left(\omega R_{0} C\right)^{2}}}
$$

$$
\begin{aligned}
& z=\left\{\left\lfloor R_{s}+R_{o}\left(1-\omega^{2} L C\right)\right\rfloor^{2}+\left\lceil\omega\left(R_{s} R_{o} C+L\right\rceil^{2}\right\}^{1 / 2}\right. \\
& \theta_{i}=\theta_{v}-\tan ^{-1}\left(\omega R_{o} C\right) \\
& \theta_{v}=\tan ^{-1}\left(\frac{\omega\left(R_{s} R_{o} C+L\right)}{R_{s}+R_{o}\left(1-\omega^{2} L C\right)}\right)
\end{aligned}
$$

La respuesta natural de $i_{s}$ tendrá un par de polos complejos conjugados, es decir, $i_{n}(t)$ es de la forma:

$$
\begin{aligned}
& i_{n}(t)=e^{-\alpha\left(t-t_{\beta}\right)}\left\{A_{1} \cos \left[\omega_{a}\left(t-t_{\beta}\right)\right]+\right. \\
& \left.A_{2} \operatorname{sen}\left[\omega_{a}\left(t-t_{\beta}\right)\right]\right\}
\end{aligned}
$$

con,

$$
\omega_{a}=\sqrt{\omega_{n}^{2}-\alpha^{2}}
$$

$t_{\beta}$ es el instante donde los diodos comienzan a conducir y se determinará posteriormente. Los coeficientes $A_{1}$ y $A_{2}$ están dados por:

$$
\begin{aligned}
& A_{1}=-V_{a} \operatorname{sen}\left(\omega t_{\beta}-\theta_{i}\right) \\
& A_{2}=\frac{1}{\omega_{a}}\left[A_{1} \alpha-V_{a} \omega \cos \left(\omega t_{\beta}-\theta_{i}\right)\right]
\end{aligned}
$$

siendo $V_{a}=V_{m} / Z_{i}$. Así, mientras conducen los diodos, la corriente que suministra la fuente es:

$$
i_{s}(t)=i_{f}(t)+i_{n}(t)
$$

con $i_{f}(t)$ e $i_{n}(t)$ dadas en (5) y (7), respectivamente.

De manera análoga, durante la conducción de los diodos del puente rectificador, el voltaje en $R_{0}$ será:

$$
v_{o}(t)=v_{f}(t)+v_{n}(t)
$$

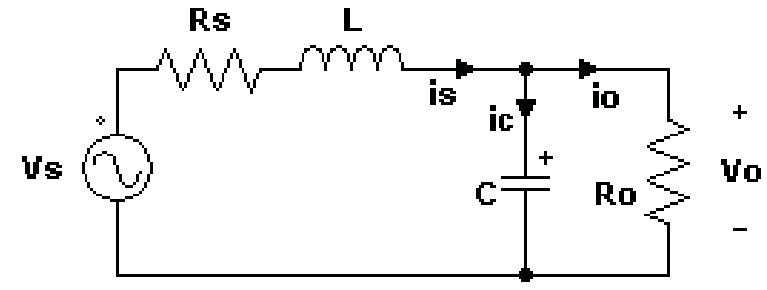

Figura 2. Circuito equivalente cuando los diodos conducen $(L=L s+L o)$ 
DOI: http://dx.doi.org/10.22201/fi.25940732e.2010.11n2.016

Contaminación armónica producida por cargas no lineales de baja potencia: modelo matemático y casos prácticos

donde, las respuestas forzada y natural son:

$$
\begin{aligned}
& v_{f}(t)=\frac{R_{o}}{z} V_{m} \operatorname{sen}\left(\omega t-\theta_{v}\right) \\
& v_{n}(t)=e^{-\alpha\left(t-t_{\beta}\right)}\left\{B_{1} \cos \left[\omega_{a}\left(t-t_{\beta}\right)\right]\right. \\
& \left.+B_{2} \operatorname{sen}\left[\omega_{a}\left(t-t_{\beta}\right)\right]\right\}
\end{aligned}
$$

Los coeficientes $B_{1}$ y $B_{2}$ están dados por:

$$
\begin{aligned}
& B_{1}=-A_{1}\left(R_{s}-\alpha L\right)-A_{2} \omega_{a} L \\
& B_{2}=A_{1} \omega_{a} L-A_{2}\left(R_{s}-\alpha L\right)
\end{aligned}
$$

Cuando los diodos salen de conducción, la etapa de entrada del circuito de la figura 1 se desconecta, el capacitor se descarga a través de $R_{0}$ y el voltaje de salida decrece en forma exponencial según la constante de tiempo $R_{0} C$, hasta que comienza a conducir el segundo par de diodos del rectificador, en el semiciclo negativo del voltaje de entrada (Hart, 2001; Mohan et al., 2003). Así, mientras los diodos no conducen, el voltaje en $R_{0}$ es:

$$
v_{0}(t)=v_{o \delta} e^{-\left(t-t_{\delta}\right) / \omega R_{0} C}
$$

$t_{\delta}$ es el instante en que se extingue la corriente de entrada $\mathrm{y}, v_{o \delta}$ es el valor del voltaje de salida en dicho instante; es decir,

$$
v_{o \delta}=v_{f}\left(t_{\delta}\right)+v_{n}\left(t_{\delta}\right)
$$

Por lo tanto, la ecuación (11) es válida en el intervalo $\left[t_{\beta}, t_{\delta}\right]$, mientras que (14) funge para $\left[t_{\delta}, t_{\beta}+T / 2\right]$, don$\operatorname{de} T=2 \pi / \omega$.

Finalmente, puesto que en el instante $t=t_{\delta}$ la corriente del suministro se hace cero, y tomando en cuenta que después de $t=t_{\beta}+\mathrm{T} / 2$ el valor del voltaje rectificado (a la salida de puente de diodos) supera al voltaje $v_{0}$ (que decae exponencialmente), $t_{\beta}$ y $t_{\delta}$ se pueden determinar al resolver el siguiente sistema de ecuaciones:

$$
\begin{aligned}
& v_{o \delta} e^{-\left[\left(t_{\beta}+T / 2\right)-t_{\delta}\right] / \omega R_{0} C}-\left|V_{m} \operatorname{sen}\left(\omega\left(t_{\beta}+T / 2\right)\right)\right|=0 \\
& i_{f}\left(t_{\delta}\right)+i_{n}\left(t_{\delta}\right)=0
\end{aligned}
$$

\section{Simulación del comportamiento de la carga no lineal}

Para llevar a cabo la simulación del circuito de la figura 1 , se ha considerado la impedancia en una línea de alimentación residencial de $120 \mathrm{~V}$ para obtener los valores de $R_{s}$ y $L_{s}$ (McInnis, 1997). A fin de conseguir un rizado pequeño en el voltaje y en la corriente de salida del rectificador, se han seleccionado valores apropiados para $L_{0}$ y $C$. Por último, $R_{0}$ se ha elegido en función de un determinado consumo de potencia. Así, se obtuvo en MATLAB la descomposición por serie de Fourier para $i_{s}(t)$ a partir de la expresión dada en (10) - tomando en cuenta también el semiciclo negativo del voltaje de entrada - con los siguientes componentes del circuito de la figura 1:

$$
\begin{array}{ll}
R_{s}=0.2 \Omega, & L_{o}=1 \mathrm{mH}, \quad R_{o}=160 \Omega, \\
\mathrm{C}=560 \mu \mathrm{F}, \quad L_{s}=160 \mu \mathrm{H}, & \\
V_{\text {rms }}=120 \mathrm{~V}, \quad f=60 \mathrm{~Hz}
\end{array}
$$

La suma de los primeros 30 componentes armónicos de la serie de Fourier resultante se muestra en la figura 3. En ella se observa que la corriente de entrada deja de ser senoidal ante una carga no lineal y muestra en este caso un comportamiento pulsante. Evidentemente, esta señal genera armónicos que se propagan a través de las líneas de suministro eléctrico, provocando problemas como calentamiento en los cables de alimentación, interferencia electromagnética, mayores demandas de potencia reactiva, etc.

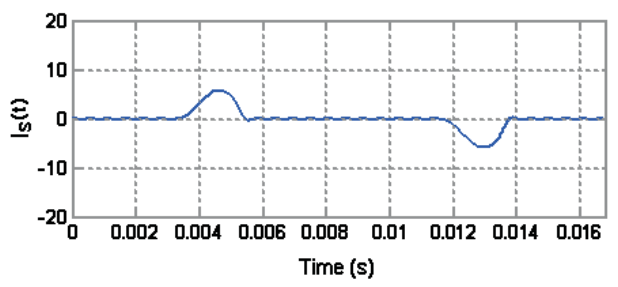

Figura 3. Aproximación de $i_{S}(t)$, por serie de Fourier

En la figura 4, se muestra la descomposición espectral, obtenida en MATLAB para la corriente suministrada a la carga no lineal. Como puede observarse, los armónicos de $i_{s}(t)$ son impares y la magnitud de los primeros es considerable en comparación con la frecuencia fundamental de $60 \mathrm{~Hz}$ (el tercer armónico, por ejemplo, es apenas un $10 \%$ más pequeño).

La figura 5 muestra las formas de onda del voltaje rectificado y filtrado, $v_{o}$, y de la corriente de suministro, $i_{s}$, obtenidas a través del simulador PSIM, considerando los mismos valores de los componentes dados arriba. La señal $\left|v_{s}\right|$, representa el valor absoluto del voltaje de entrada, $v_{s}$. 
DOI: http://dx.doi.org/10.22201/fi.25940732e.2010.11n2.016

Mora-Barajas M.A. y Bañuelos-Sánchez P.

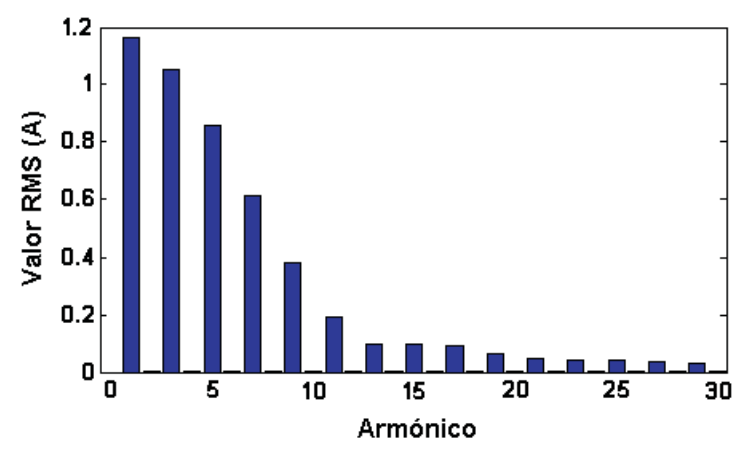

Figura 4. Descomposición espectral de $i_{s}(t)$

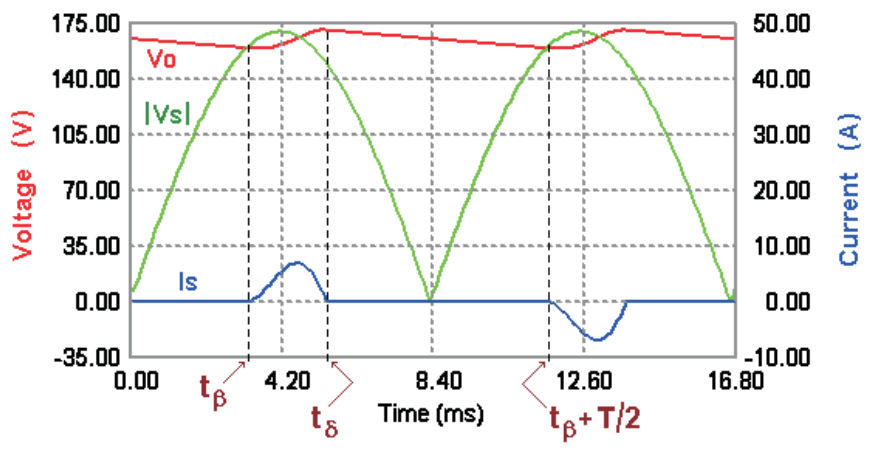

Figura 5. Voltaje en la carga y corriente de suministro en el circuito de la figura 1

\section{Armónicos medidos en cargas residenciales}

Se realizó la medición de los armónicos de una variedad de cargas residenciales de diferentes marcas, modelos y tecnologías, todas ellas con una antigüedad menor a cinco años. Dichas mediciones, se realizaron conforme lo establece la norma IEC 1000-3-2 (referente a los límites permisibles para armónicos de corriente en aquellos aparatos que consumen menos de $16 \mathrm{~A}$ por fase), utilizando el analizador de armónicos Fluke 41. En los resultados que a continuación se presentan, se muestra el efecto de más de una carga conectada, aún cuando se hicieron también las mediciones individuales para la mayoría de los aparatos, pues el efecto de la contaminación armónica en las líneas de alimentación alterna se hace importante no por una carga individual, sino por la combinación simultánea de varias de ellas.

La figura 6 muestra la corriente de alimentación medida al estar encendidos simultáneamente 4 televisores a color, de 14", 20", 20" y 21". Su forma de onda es muy similar a la mostrada en la figura 3 . En las tablas de la figura 6 se puede observar, por ejemplo, que la magnitud RMS del tercer armónico es de 1.23A. Tomando en cuenta que la corriente total RMS suministrada es de
2.26A, la amplitud de este armónico es tan grande como un $54.68 \%$ de la corriente RMS total. La distorsión armónica total (THD) es del $70.38 \%$ en la corriente y $1.81 \%$ en el voltaje. El factor de potencia $\mathrm{PF}=0.7$, tienen en conjunto un consumo de potencia de $200 \mathrm{~W}$. Si la corriente fuese una senoidal pura tendría un $\mathrm{THD}=0$ y si estuviese en fase con el voltaje de línea, su factor de potencia debería ser unitario $(\mathrm{PF}=1)$. Un $\mathrm{PF}<1$ incrementa los requerimientos de potencia reactiva del equipo, elevando los costos tanto para el consumidor como para el proveedor del servicio (Chen, 1990).

Al conectar un televisor junto con un reproductor de DVD o una videograbadora, se modifican ligeramente las intensidades de los armónicos, pero la corriente conserva una forma de onda similar a la que se presenta en la figura 6.

Los equipos modulares o mini-componentes, al igual que los televisores y demás equipos ya mencionados, cuentan con un rectificador de entrada; sin embargo, en la forma de onda de la corriente de varios mini-componentes se encontró un menor contenido armónico, en comparación con la figura 6 , la fundamental tiene mayor amplitud, y por tanto, los armónicos son de menor intensidad. 

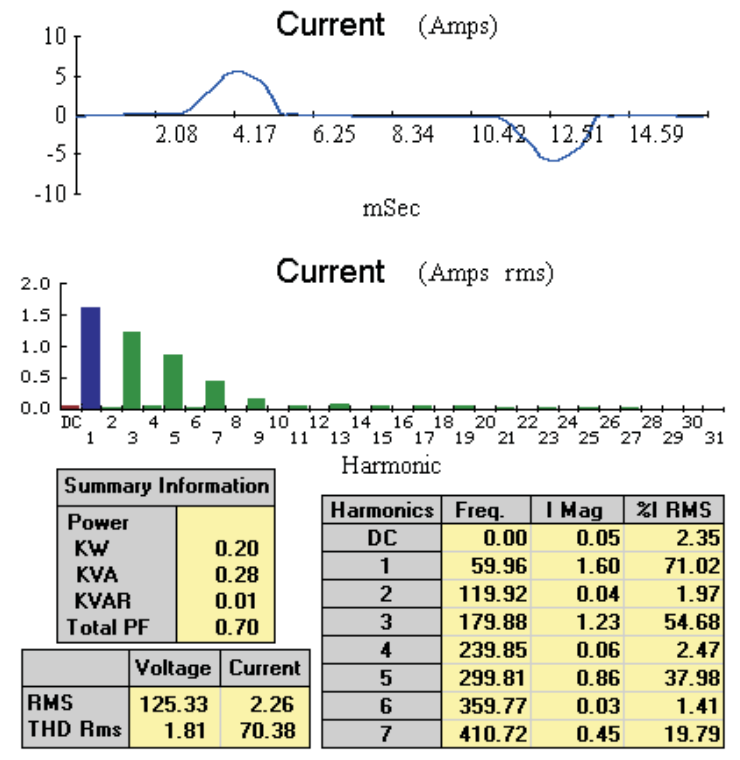

Figura 6. Corriente consumida por 4 televisores operando simultáneamente

La figura 7 muestra las características en la corriente que demanda una computadora personal (PC), que cuenta con un monitor LCD de 17", conectada directamente a la línea alterna. La forma de onda es pulsante, al igual que en el caso de los televisores. De acuerdo con la adaptación que la Asociación Canadiense para la Estandarización, CSA, realizó a la norma IEC 1000-3-2, para el caso de las líneas de $120 \mathrm{Vrms} / 60 \mathrm{~Hz}$ (McInnis, 1997), el valor máximo para el tercer armónico de la corriente en un aparato que consume menos de 600W, debe ser de $5.54 \mathrm{~mA} / \mathrm{W}$. Tomando en cuenta que esta PC consume $100 \mathrm{~W}$, el tercer armónico debería ser menor a $0.554 \mathrm{~A}$; sin embargo, de acuerdo con las mediciones mostradas en las tablas de la figura 7 , el valor rms de este armónico es de 0.71A. Los armónicos 5 y 7 , cuyos valores respectivos son 0.51 y $0.3 \mathrm{~A}$ (según los datos de la figura 7), respectivamente, deberían ser menores a 3.51 y $1.95 \mathrm{~mA} / \mathrm{W}$ (lo que resulta en 0.351 y $0.195 \mathrm{~A}$ ) de acuerdo con la CSA.
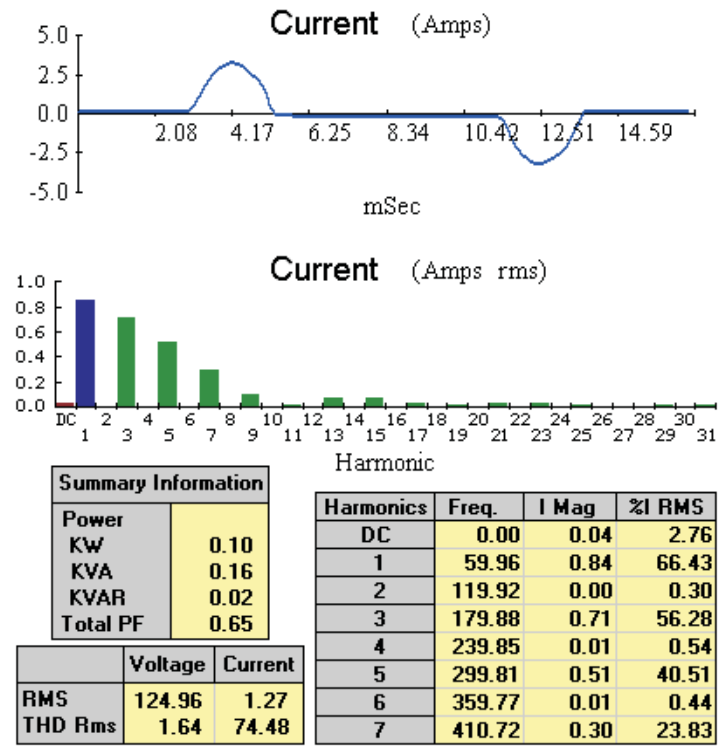

Figura 7. Corriente demandada por una PC 
Resultados similares a los mostrados en la figura 7 se obtuvieron con computadoras personales conectadas a la línea, a través de fuentes reguladoras de voltaje, tanto con monitores de CRT como de LCD.

Uno más de los elementos considerados en este trabajo, son las lámparas fluorescentes. Estas lámparas dan mayor luminosidad con un menor consumo de potencia eléctrica, pero tienen un aporte importante en la contaminación armónica. En la figura 8 se muestra la corriente que demandan al estar encendidas simultáneamente tres lámparas fluorescentes de balastro electrónico, del mismo modelo y marca, cuya especificación individual de consumo de potencia es de $20 \mathrm{~W}$.

La forma de onda de la corriente es completamente diferente al resto de los equipos ya mencionados y tiene una nueva característica, la aparición del segundo armónico, que en la medición individual de algunas lámparas llega a tener una magnitud similar a la de sus armónicos 5 o 7 .

Las lámparas fluorescentes que cuentan con un balastro electromagnético, tienen un muy bajo factor de potencia, pero su contenido armónico es mínimo, en comparación con las de balastro electrónico, ya que representan más bien una carga del tipo inductivo.

En las figuras 9 y 10 se muestra el efecto combinado de varias cargas operando de manera simultánea en un hogar. La figura 9 corresponde a un televisor a color de 14 " y un equipo modular estéreo de $60 \mathrm{~W}$ de potencia especificada. En tanto que la figura 10 muestra la corriente que demandan conjuntamente un televisor a color de
20", una PC, tres lámparas fluorescentes (con una potencia nominal de $22 \mathrm{~W}$ cada una) y un mini-componente, cuya potencia nominal máxima es de 190W. En ambos casos, los mini-componentes reproducían la música de un $\mathrm{CD}$ a un volumen moderado.

La distorsión armónica total en la corriente es del $37.01 \%$ en el caso de la figura 9 , y del $55.54 \%$ en el caso de la figura 10. Como se aprecia en ambos casos, al conectar conjuntamente varias cargas combinadas se producen niveles de contaminación armónica más bajos que el que genera cada carga individualmente.

Esto es debido a que el ángulo de fase es diferente para un mismo armónico en cada carga y el contenido de armónicos resultante es la suma de las corrientes armónicas individuales.

Las cargas residenciales como refrigeradores y lavadoras que poseen algún tipo de motor, producen también contaminación armónica pero, puesto que la corriente que demandan sus motores es mucho mayor que la de sus partes electrónicas, su comportamiento es más bien relacionado al de una carga inductiva.

De manera similar ocurrió al medir cargas como las impresoras láser, que presentan un comportamiento de tipo resistivo cuando están imprimiendo.

Por otro lado, cabe resaltar que dentro de las mediciones realizadas, además de un bajo factor de potencia se encontró también que algunas cargas residenciales tienen una importante demanda de potencia reactiva; por ejemplo, las cargas combinadas del caso de la figura 10 tienen un consumo de potencia activa de $280 \mathrm{~W}$ y
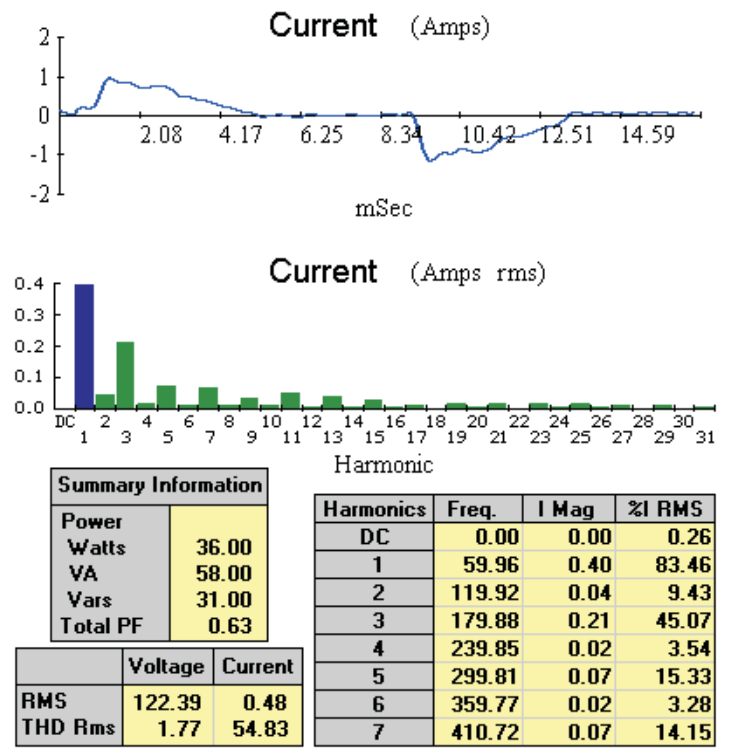

Figura 8. Corriente consumida por 3 lámparas fluorescentes con balastro electrónico 

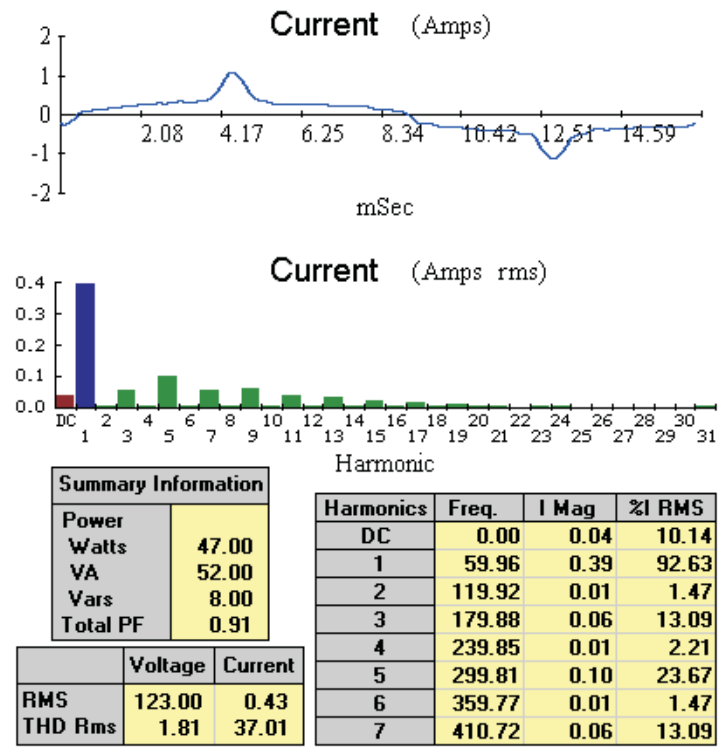

Figura 9. Corriente consumida por una TV de 14" (a color) junto con un mini-componente
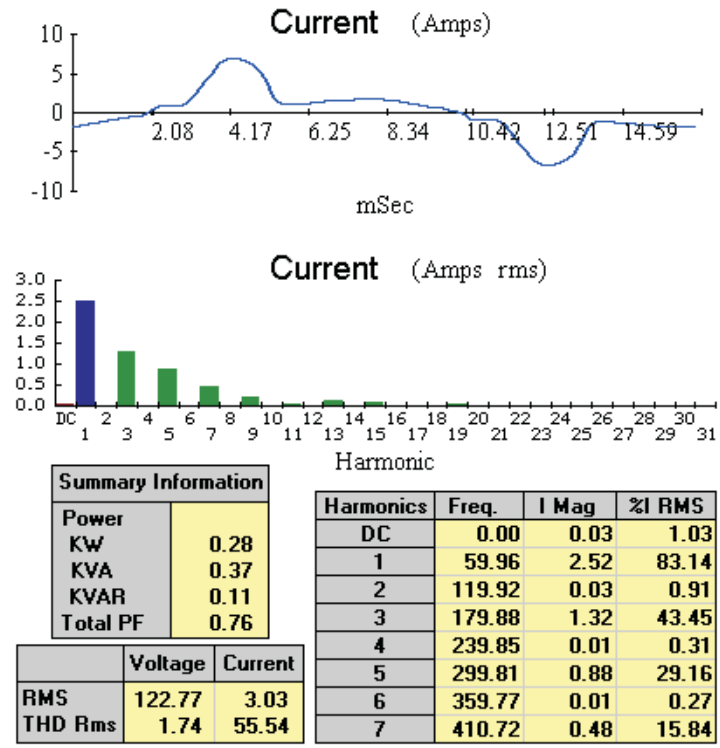

Figura 10. Corriente que demandan varias cargas residenciales, simultáneamente

demandan una potencia reactiva de 110VAR. Si bien, la potencia reactiva no es consumida por los equipos eléctricos, si es necesario suministrarla para que funcionen. Entre mayor sea la demanda de potencia reactiva, se requerirá una mayor generación de energía eléctrica y conductores de mayor calibre para su distribución, haciendo que los costos de producción, distribución y consumo sean más altos.

Es importante destacar que, de acuerdo con la información de las tablas en las figuras 6-10, la distorsión armónica total en el voltaje del suministro eléctrico es menor al 2\%, lo que significa que la forma de onda de la línea de CA es prácticamente senoidal, por lo que los armónicos medidos en la corriente de los diferentes equipos probados, son generados por los equipos mismos.

Finalmente, cabe mencionar que con el simple hecho de estar conectados (sin estar encendidos), los electrodomésticos consumen energía. Por ejemplo, las 4 televisiones del caso de la figura 6 consumen en conjunto $19 \mathrm{~W}$, en tanto que cada mini-componente consume 
más de $15 \mathrm{~W}$, es como tener "una lámpara eternamente encendida", que impacta directamente sobre la economía familiar y representa un gasto infructuoso de energía.

\section{Conclusiones}

Hasta hoy, la mayor parte de los esfuerzos por regular los límites de contaminación armónica se han llevado a cabo para las cargas industriales; sin embargo, el problema también existe en las líneas de distribución residencial, aunque quizás, aún no se ha medido el efecto perjudicial del fenómeno.

Muy a pesar de la existencia de normas que establecen límites para el contenido armónico en la corriente demandada por las cargas eléctricas, el estudio realizado en este trabajo demostró que los aparatos electrónicos más utilizados en los hogares, generan altos niveles de contaminación armónica sobre las líneas de distribución eléctrica.

Una gran preocupación de las compañías generadoras de electricidad (específicamente la CFE en México) sigue siendo reducir el consumo de energía en los hogares. Si las fuentes internas de los aparatos electrónicos se diseñan empleando convertidores que demanden corrientes de tipo senoidal, con un alto factor de potencia (algunas veces también llamados convertidores limpios), se lograría no solo reducir la contaminación armónica introducida hacia las líneas de suministro eléctrico, sino que también permitiría reducir el consumo de potencia, que se traduce en un beneficio, tanto para los productores de electricidad como para los usuarios.

Por último, es muy importante atender la recomendación de mantener desconectado todo electrodoméstico cuando no está siendo utilizado. Sin importar que estén apagados, la potencia que estos equipos consumen al estar conectados de ninguna manera puede considerarse una cantidad despreciable, pues representa un importante gasto energético infructuoso. Su impacto no solo es económico sino también ambiental, pues la mayoría de los procesos utilizados actualmente para generar energía eléctrica, contribuyen de manera importante a la contaminación del medio ambiente.

\section{Referencias}

Abad-San Martín V., González de la Viuda J., Sagredo-González J. Influencia de cargas no lineales en transformadores de distribución. $8^{\circ}$ Reunión Nacional de Grupos de Investigación en Ingeniería Eléctrica. Cádiz, Andalucía, España. Enero, 1998.

Arrillaga J., Bradley D.A., Bodger P.S. Power System Harmonics. John Wiley \& Sons. Norwich. U.K. 1985. Pp. 71-75.

Brugnoni M. Los componentes armónicos de la demanda y sus efectos sobre las redes de distribución eléctricas [en línea]. 2003. Disponible en:

www.fi.uba.ar/materias/6509/Art\%EDculos\%20Arm\%F 3nicos/Paper\%20GEA\%20Arm\%F3nicos\%20en\%20redes. doc

Chen K. Industrial Power Distribution and Illuminating Systems. Marcel Dekker, Inc. USA. 1990. Pp. 199-208.

Eguíluz L., Lavandero J., Mañana M., Sánchez P. Eficiencia energética y calidad de suministro eléctrico. Revista: ENERGÍA. Madrid, Marzo, 2001.

Hart D. Electrónica de potencia. Pearson Education. Madrid. 2001. Pp. 88-94.

Hibbard J.F., Lowenstein M. Meeting IEEE 519-1992 Harmonic limits. Technical papers, Trans-coil, Inc. [en línea]. 1995. Disponible en: http://www.transcoil.com/meetieee.pdf

IEC 1000-3-2. Límites para la emisión de corrientes armónicas en los aparatos que consumen menos de $16 \mathrm{~A}$ por fase. 1995.

Jiménez R., Cerero J. Perspectiva de calidad de la energía eléctrica y su importancia en México. Encuentro Nacional de Metrología Eléctrica, Querétaro, México, junio, 2005.

Mohan N., Undeland T., Robbins W. Power Electronics. Converters, applications and design. John Wiley \& Sons. Third edition. USA. 2003. Pp. 95-98.

McInnis M. Low Voltage, Low Frequency Harmonic Emission Limits [en línea]. 1997. Disponible en:

http://grouper.ieee.org/groups/harmonic/single/docs/can ada.doc

Suárez J., Mauro G., Anault D., Agüero C. Análisis de la distorsión armónica y los efectos de atenuación y diversidad en áreas residenciales. Revista del IEEE América Latina, 3(5):71-77, diciembre, 2005. 
DOI: http://dx.doi.org/10.22201/fi.25940732e.2010.11n2.016

Contaminación armónica producida por cargas no lineales de baja potencia: modelo matemático y casos prácticos

\section{Semblanzas de los autores}

Marco Antonio Mora-Barajas. Realizó su licenciatura en electrónica en la Benemérita Universidad Autónoma de Puebla (BUAP). En 2008, concluyó sus estudios de maestría en ciencias con especialidad en ingeniería electrónica en la Universidad de las Américas Puebla. Su área de interés es la electrónica de potencia con especial atención en el diseño de correctores de factor de potencia monofásicos y trifásicos. Publicó un artículo en el Congreso Internacional en Electrónica, Comunicaciones y Computadoras (CONIELECOMP).

Pedro Bañuelos-Sánchez. Obtuvo su doctorado en ingeniería eléctrica con especialidad en electrónica de potencia por la Universidad Paris VI-Escuela Superior de Electricidad (SUPELEC) en Francia. Su área de especialidad se encuentra enfocada en el diseño y aplicación de convertidores estáticos de potencia, como los convertidores CD/CD, CD/CA y CA/CA. Fue investigador visitante en la Universidad Picardie Jules Verne, en Amiens Francia en 2003. También trabajó como investigador asociado por parte del CNRS (Centre National de la Recherche Scientifique) en el Laboratoire d'Electrotechnique de Grenoble, en Grenoble Francia en 2006. Trabajó como instructor del curso Principios de Diseño en Ingeniería para Johns Hopkins University Center for Talented Youth en 2008. Ha participado como sinodal externo en tesis de doctorado en universidades del extranjero, tales como la Universidad Politécnica de Cataluña (marzo de 2007) y la Universidad Paris XI (septiembre de 2007); así también en universidades nacionales tales como el Centro Nacional de Investigación y Desarrollo Tecnológico (CENIDET) y el Instituto Politécnico Nacional (IPN). Actualmente trabaja en la Universidad de las Américas Puebla, donde ha sido galardonado con el premio a la docencia en 2003 y 2004. Ha sido revisor técnico en diversos congresos internacionales y nacionales, así como en revistas en el área de electrónica de potencia. También fue presidente del comité organizador del Congreso Internacional de Electrónica de Potencia (CIEP). Es miembro del IEEE Power Electronics Society y de la Sociedad Mexicana en Electrónica de Potencia (SOMEP). 